\title{
Use of the canonical approach in effective models of QCD
}

\author{
Masayuki Wakayama $\oplus^{1,2,3,4,{ }^{*}}$ Seung-il Nam๑ ${ }^{1,2,5}$ and Atsushi Hosaka $\oplus^{3,6}$ \\ ${ }^{1}$ Department of Physics, Pukyong National University (PKNU), Busan 48513, Republic of Korea \\ ${ }^{2}$ Center for Extreme Nuclear Matters (CENuM), Korea University, Seoul 02841, Republic of Korea \\ ${ }^{3}$ Research Center for Nuclear Physics (RCNP), Osaka University, Ibaraki, Osaka 567-0047, Japan \\ ${ }^{4}$ School of Science and Engineering, Kokushikan University, Tokyo 154-8515, Japan \\ ${ }^{5}$ Asia Pacific Center for Theoretical Physics (APCTP), Pohang 790-784, Republic of Korea \\ ${ }^{6}$ Advanced Science Research Center, Japan Atomic Energy Agency (JAEA), Tokai 319-1195, Japan
}

(Received 6 April 2020; accepted 3 August 2020; published 28 August 2020)

\begin{abstract}
We discuss the canonical approach for the study of QCD phase at finite densities and temperatures in the confinement phase. The canonical approach, which is a method to extrapolate observables calculated at pure imaginary chemical potentials to those at real chemical potentials, is useful to overcome the sign problem in lattice QCD simulations at finite density. To validate the applicability of the approach, we employ the Nambu-Jona-Lasinio (NJL) and Polyakov-NJL (PNJL) models where exact solutions for the number density are available, which is the basic input of the fugacity expansion and can be compared with those of the canonical approach. We find that the number densities computed from the canonical approach are consistent with the exact solutions in most of the confinement phase. The results in the present study are applicable to the study of lattice QCD.
\end{abstract}

DOI: 10.1103/PhysRevD.102.034035

\section{INTRODUCTION}

Understanding of quantum chromodynamics (QCD) at finite temperature and density has been highly demanded as fundamental inputs for the studies of various interesting questions such as the generation of matter in the early universe, the galaxy formations and mysterious stellar objects such as neutron stars and black holes. The high energy accelerators at such as J-PARC (KEK/JAEA), FAIR (GSI) and NICA (JINR) will be expected to operate in the near future to approach these questions. In the theoretical side, lattice QCD is an almost unique method for the first principle approach to QCD.

As already well known, however, lattice QCD simulations at finite density suffer from the sign problem due to a complex value of the grand canonical partition function. To attack this problem, the canonical approach has been proposed [1], and has been developed rapidly by using multiple-precision arithmetic [2-18]. In the canonical approach, lattice QCD simulations are performed at pure imaginary chemical potentials where the grand canonical partition function is real, that overcomes the sign problem.

\footnotetext{
*wakayama@rcnp.osaka-u.ac.jp
}

Published by the American Physical Society under the terms of the Creative Commons Attribution 4.0 International license. Further distribution of this work must maintain attribution to the author(s) and the published article's title, journal citation, and DOI. Funded by SCOAP ${ }^{3}$.
The canonical approach can be applied to the study of physical observables such as particle number distributions in heavy-ion collisions and of the phase structure at the chemical potential. Despite these advantages, there remains a question of accuracy of the method when only limited amount of lattice data are available.

In this paper, we would like to address this question by using QCD effective models such as the Nambu-JonaLasinio (NJL) and Polyakov-NJL (PNJL) ones where exact solutions are known in principle. The advantage of the models is that it is possible to perform (semi) analytically the canonical approach.

The NJL model has been successful in describing various properties of nonperturbative QCD [19-22]. In our previous paper [18], the model was applied to the LeeYang zero method for the QCD phase structure. The PNJL model incorporates not only spontaneous symmetry breaking of chiral symmetry but also the spontaneous breaking of $Z\left(N_{c}\right)$ center symmetry. The latter is governed by the expectation value of the Polyakov loop $\langle\Phi\rangle$ as an order parameter for confinement and deconfinement phases $[23,24]$. In this way, the PNJL model incorporates an important feature of the gluon dynamics of QCD.

Our strategy is as follows. In accordance with the lattice data analyses, first, we compute the quark number density at pure imaginary chemical potentials in the effective models. The resulting quark number density as a function of the chemical potential is parametrized by a Fourier series of a finite number of terms $N_{\sin }$. The validity of the 
canonical approach is determined by the accuracy of the parametrization, the investigation of which is the main subject of the present paper. Furthermore, we introduce the maximum value of fluctuations of the net quark number $N_{\max }$ that is needed in lattice simulations due to finite amounts of resources. A comparison of the results of finite $N_{\max }$ with the exact ones also provides a measure of the validity of the canonical approach in the actual lattice simulations.

From the numerical results, we find that the canonical approach works qualitatively well even near the phasetransition line for relatively small values of $N_{\max }$ and $N_{\mathrm{sin}}$, $N_{\max } / V \gtrsim 0.56\left[\mathrm{fm}^{-3}\right]$ and $N_{\sin } \approx 4$, where $V$ is a volume in the system. Especially, $N_{\text {sin }}=1$ or 2 is enough to reconstruct the number density within $10 \%$ accuracy for the temperature below one of the critical endpoint and for the baryon chemical potential $<900[\mathrm{MeV}]$.

The present paper is organized as follows: In Sec. II, we briefly explain the canonical approach in the PNJL model. The numerical results are given in Sec. III with detailed discussions. Section IV is devoted to summary and future perspectives.

\section{THE CANONICAL APPROACH IN THE PNJL MODEL}

\section{A. The canonical approach}

In this subsection, we review the canonical approach. Let us start with the relation between the grand canonical partition function $Z_{G C}$ and the canonical partition functions $Z_{C}$ as a fugacity expansion,

$$
Z_{\mathrm{GC}}(\mu, T, V)=\sum_{n=-\infty}^{\infty} Z_{C}(n, T, V) \xi^{n},
$$

where $\mu, T, V$ and $\xi\left(\equiv e^{\mu / T}\right)$ are the quark chemical potential, temperature, volume of the system and the quark fugacity, respectively. The canonical partition function is computed by the Fourier transform of Eq. (1) over the imaginary chemical potential,

$$
Z_{C}(n, T, V)=\int_{-\pi}^{\pi} \frac{d \theta}{2 \pi} e^{-i n \theta} Z_{\mathrm{GC}}\left(\mu=i \mu_{I}, T, V\right),
$$

where $\mu_{I}$ is real and $\theta=\mu_{I} / T$. Because the Fourier transform has cancellations of significant digits that come from the high frequency part of $e^{-i n \theta}$ at large $n$, multipleprecision arithmetic is needed in numerical calculations.

The integration method is also used to extract $Z_{C}$ for larger $n$ in lattice QCD calculations [13-17]. In the integration method, $Z_{G C}\left(i \mu_{I}\right)$ in Eq. (2) is derived from the number density at the pure imaginary chemical potential,

$$
\frac{n_{q}}{T^{3}}\left(i \mu_{I}\right)=\frac{1}{V T^{2}} \frac{\partial}{\partial\left(i \mu_{I}\right)} \ln Z_{G C}\left(i \mu_{I}\right) .
$$

Because $Z_{G C}\left(i \mu_{I}\right)$ is real, we can define as $n_{q}\left(i \mu_{I}\right)=i n_{q I}$ with the real valued $n_{q I}$. The imaginary number density $n_{q I}$ is approximated by a Fourier series,

$$
\frac{n_{q I}}{T^{3}}(\theta)=\sum_{k=1}^{N_{\text {sin }}} f_{k} \sin (k \theta),
$$

with a finite number of terms of $N_{\sin }$ [25-27]. After getting a set of coefficients $f_{k}$, we can evaluate $Z_{G C}\left(i \mu_{I}\right)$ in good approximation from

$$
\begin{aligned}
Z_{\mathrm{GC}}\left(i \mu_{I}, T, V\right) & =C \exp \left[-V \int_{0}^{\theta} d \theta^{\prime} n_{q I}\left(\theta^{\prime}\right)\right] \\
& =C \exp \left[V T^{3} \sum_{k=1}^{N_{\text {sin }}} \frac{f_{k}}{k} \cos (k \theta)\right],
\end{aligned}
$$

where $C$ is an integration constant.

\section{B. The PNJL model}

The effective potential $\omega$ of the PNJL model is given as

$$
\begin{aligned}
\omega= & \frac{1}{2 G}\left(M-m_{q}\right)^{2}-2 N_{c} N_{f} \int \frac{d^{3} p}{(2 \pi)^{3}} E_{p} \\
& -2 N_{f} T \int \frac{d^{3} p}{(2 \pi)^{3}}\left\{\operatorname{Tr}_{c} \ln \left[1+L e^{-\frac{E_{p}-\mu}{T}}\right]\right. \\
& \left.+\operatorname{Tr}_{c} \ln \left[1+L^{\dagger} e^{-\frac{E_{p}+\mu}{T}}\right]\right\}+\omega_{g},
\end{aligned}
$$

where the energy and the constituent quark mass are defined by $E_{p}=\sqrt{p^{2}+M^{2}}$ and $M=m_{q}-G \sigma$, respectively, with the current quark mass $m_{q}$, the coupling constant $G$ and the chiral condensate $\sigma$. The Polyakov loop $L$ is defined by

$$
L(\vec{x})=\mathcal{P} \exp \left[i \int_{0}^{1 / T} d x_{4} A_{4}\left(\vec{x}, x_{4}\right)\right],
$$

where $\mathcal{P}$ stands for the path ordering and $A_{4}=i A_{0}$ is the $S U\left(N_{c}\right)$ temporal-gauge field in Euclidian space. Moreover, we express the polynomial Polyakov-loop potential as the gauge-field contribution of the effective potential,

$\omega_{g}(T, \mu)=T^{4}\left[-\frac{b_{2}(T)}{2} \ell \bar{\ell}-\frac{b_{3}}{6}\left(\ell^{3}+\bar{\ell}^{3}\right)+\frac{b_{4}}{4}(\ell \bar{\ell})^{2}\right]$,

where $\ell$ and $\bar{\ell}$ are the thermal expectation values of the color trace of the Polyakov loop and its conjugate,

$$
\ell(\vec{x}) \equiv \frac{1}{N_{c}}\left\langle\operatorname{Tr}_{c} L(\vec{x})\right\rangle, \quad \bar{\ell}(\vec{x}) \equiv \frac{1}{N_{c}}\left\langle\operatorname{Tr}_{c} L^{\dagger}(\vec{x})\right\rangle .
$$

Note that $\operatorname{Tr}_{c} L$ and $\operatorname{Tr}_{c} L^{\dagger}$ are generally complex in $S U\left(N_{c}\right)$ for $N_{c} \geq 3$. We choose the parameters in Eq. (8) as in Ref. [28]: 
$b_{2}(T)=a_{0}+a_{1}\left(\frac{T_{0}}{T}\right)+a_{2}\left(\frac{T_{0}}{T}\right)^{2}+a_{3}\left(\frac{T_{0}}{T}\right)^{3}$,

$a_{0}=6.75, \quad a_{1}=-1.95, \quad a_{2}=2.625, \quad a_{3}=-7.44$, $b_{3}=0.75, b_{4}=7.5$ and $T_{0}=270[\mathrm{MeV}]$.

For $N_{c}=3$, Polyakov loops are represented as $L=\operatorname{diag}\left(e^{i \varphi_{1}}, e^{i \varphi_{2}}, e^{-i\left(\varphi_{1}+\varphi_{2}\right)}\right)$ in the Polyakov gauge. Therefore, we can rewrite the color traces in Eq. (6) as follows,

$$
\begin{aligned}
& \operatorname{Tr}_{c} \ln \left[1+L e^{-\frac{E_{p}-\mu}{T}}\right] \\
&= \ln \left[1+\operatorname{Tr}_{c} L e^{-\frac{E_{p}-\mu}{T}}+\operatorname{Tr}_{c} L^{\dagger} e^{-\frac{2\left(E_{p}-\mu\right)}{T}}+e^{-\frac{3\left(E_{p}-\mu\right)}{T}}\right] \\
& \rightarrow \ln \left[1+3 \ell e^{-\frac{E_{p}-\mu}{T}}+3 \bar{\ell} e^{-\frac{2\left(E_{p}-\mu\right)}{T}}+e^{-\frac{3\left(E_{p}-\mu\right)}{T}}\right],
\end{aligned}
$$

$$
\begin{aligned}
\operatorname{Tr}_{c} & \ln \left[1+L^{\dagger} e^{-\frac{E_{p}+\mu}{T}}\right] \\
= & \ln \left[1+\operatorname{Tr}_{c} L^{\dagger} e^{-\frac{E_{p}+\mu}{T}}+\operatorname{Tr}_{c} L e^{-\frac{2\left(E_{p}+\mu\right)}{T}}+e^{-\frac{3\left(E_{p}+\mu\right)}{T}}\right] \\
& \rightarrow \ln \left[1+3 \bar{\ell} e^{-\frac{E_{p}+\mu}{T}}+3 \ell e^{-\frac{2\left(E_{p}+\mu\right)}{T}}+e^{-\frac{3\left(E_{p}+\mu\right)}{T}}\right],
\end{aligned}
$$

where we replace $\operatorname{Tr}_{c} L$ and $\operatorname{Tr}_{c} L^{\dagger}$ by $\ell$ and $\bar{\ell}$ in the mean field approximation in the third lines of each equation. The values of $\ell, \bar{\ell}$ and $\sigma$ are the solutions of the gap equations that are nothing but the three stationary conditions:

$$
\frac{\partial \omega}{\partial \sigma}=0, \quad \frac{\partial \omega}{\partial \ell}=0, \quad \frac{\partial \omega}{\partial \bar{\ell}}=0 .
$$

\section{The PNJL model at the pure imaginary chemical potential}

In this paper, we compute $n_{q I}$ in Eq. (4) in the PNJL model. Practically, it is convenient to evaluate $n_{q I}$ numerically by

$$
\begin{aligned}
n_{q I}\left(\mu_{I}\right) & =\frac{1}{T} \frac{\partial \omega}{\partial\left(\mu_{I} / T\right)} \\
& \approx \frac{\omega\left(\mu_{I} / T+\delta\left(\mu_{I} / T\right)\right)-\omega\left(\mu_{I} / T-\delta\left(\mu_{I} / T\right)\right)}{2 T \delta\left(\mu_{I} / T\right)},
\end{aligned}
$$

where we use $\delta\left(\mu_{I} / T\right)=10^{-18}$. The calculations of $n_{q I}$ are carried out with 128 significant digits in decimal notation by using a multiple-precision arithmetic package, FMLIB [29].

For pure imaginary chemical potentials, $\ell$ and $\bar{\ell}$ are complex conjugate to each other, $\ell \equiv \ell_{r} e^{i \ell_{\phi}}, \bar{\ell}=\ell^{\dagger}=$ $\ell_{r} e^{-i \ell_{\phi}}$, where $\ell_{r}$ and $\ell_{\phi}$ are real. Therefore, $\omega\left(\mu_{I} / T\right)$ is obtained from the three stationary conditions:

$$
\frac{\partial \omega}{\partial \sigma}=0, \quad \frac{\partial \omega}{\partial \ell_{r}}=0, \quad \frac{\partial \omega}{\partial \ell_{\phi}}=0 .
$$

The resulting three gap equations are as follows:

$$
\begin{aligned}
& M=m_{q}+\frac{3 N_{f} G M}{\pi^{2}} \int_{0}^{\Lambda} d p \frac{p^{2}}{E_{p}} \\
& \times\left[1-\frac{\ell e^{-\frac{E_{p}-i \mu_{I}}{T}}+2 \ell^{*} e^{-\frac{2\left(E_{p}-i \mu_{I}\right)}{T}}+e^{-\frac{3\left(E_{p}-i \mu_{I}\right)}{T}}}{1+3 \ell e^{-\frac{E_{p}-i \mu_{I}}{T}}+3 \ell^{*} e^{-\frac{2\left(E_{p}-i \mu_{I}\right)}{T}}+e^{-\frac{3\left(E_{p}-i \mu_{I}\right)}{T}}}\right. \\
& \left.-\frac{\ell^{*} e^{-\frac{E_{p}+i \mu_{I}}{T}}+2 \ell e^{-\frac{2\left(E_{p}+\mu_{I}\right)}{T}}+e^{-\frac{3\left(E_{p}+i \mu_{I}\right)}{T}}}{1+3 \ell^{*} e^{-\frac{E_{p}+i_{I}}{T}}+3 \ell e^{-\frac{2\left(E_{p}+i \mu_{I}\right)}{T}}+e^{-\frac{3\left(E_{p}+i \mu_{I}\right)}{T}}}\right], \\
& \ell_{r}=\frac{1}{b_{2}(T)}\left[-b_{3} \ell_{r}^{2} \cos \left(3 \ell_{\phi}\right)+b_{4} \ell_{r}^{3}-\frac{3 N_{f}}{\pi^{2} T^{3}} \int_{0}^{\Lambda} d p p^{2}\right. \\
& \times\left\{\frac{e^{i \ell_{\phi}} e^{-\frac{E_{p}-i \mu_{I}}{T}}+e^{-i \ell_{\phi}} e^{-\frac{2\left(E_{p}-i \mu_{I}\right)}{T}}}{1+3 \ell e^{-\frac{E_{p}-i \mu_{I}}{T}}+3 \ell^{*} e^{-\frac{2\left(E_{p}-i \mu_{I}\right)}{T}}+e^{-\frac{3\left(E_{p}-i \mu_{I}\right)}{T}}}\right. \\
& \left.\left.+\frac{e^{-i \ell_{\phi}} e^{-\frac{E_{p}+i \mu_{I}}{T}}+e^{i \ell_{\phi}} e^{-\frac{2\left(E_{p}+\mu_{I}\right)}{T}}}{1+3 \ell^{*} e^{-\frac{E_{p}+i \mu_{I}}{T}}+3 \ell e^{-\frac{2\left(E_{p}+i \mu_{I}\right)}{T}}+e^{-\frac{3\left(E_{p}+i \mu_{I}\right)}{T}}}\right\}\right], \\
& \sin \left(\ell_{\phi}\right)=\frac{4}{3} \sin ^{3}\left(\ell_{\phi}\right)+\frac{i N_{f}}{\pi^{2} b_{3} \ell_{r}^{3} T^{3}} \int_{0}^{\Lambda} d p p^{2} \\
& \times\left[\frac{\ell e^{-\frac{E_{p}-i \mu_{I}}{T}}-\ell^{*} e^{-\frac{2\left(E_{p}-i \mu_{I}\right)}{T}}}{1+3 \ell e^{-\frac{E_{p}-i \mu_{I}}{T}}+3 \ell^{*} e^{-\frac{2\left(E_{p}-i \mu_{I}\right)}{T}}+e^{-\frac{3\left(E_{p}-i \mu_{I}\right)}{T}}}\right. \\
& \left.+\frac{-\ell^{*} e^{-\frac{E_{p}+i \mu_{I}}{T}}+\ell e^{-\frac{2\left(E_{p}+i \mu_{I}\right)}{T}}}{1+3 \ell^{*} e^{-\frac{E_{p}+i \mu_{I}}{T}}+3 \ell e^{-\frac{2\left(E_{p}+i \mu_{I}\right)}{T}}+e^{-\frac{3\left(E_{p}+i_{I}\right)}{T}}}\right] .
\end{aligned}
$$

Note that $M$ is real for pure imaginary chemical potentials. We take $N_{f}=2, m_{q}=5.5[\mathrm{MeV}], G=0.214\left[\mathrm{fm}^{2}\right]$ and the tree-momentum cutoff $\Lambda=631[\mathrm{MeV}]$, respectively, which are fixed to reproduce the pion decay constant $f_{\pi}=$ $93[\mathrm{MeV}]$ and the constituent quark mass $M=335[\mathrm{MeV}]$ in the mean field approximation.

\section{NUMERICAL RESULTS}

\section{A. Exact results in the PNJL model}

Figure 1 shows the exact result of the real baryon number density $n_{B}=n_{q} / 3$ as a function of temperature and baryon chemical potential $\left(\mu_{B}=3 \mu\right)$ in the PNJL model. The critical end point $(\mathrm{CEP}):\left(T^{\mathrm{CEP}}, \mu_{B}^{\mathrm{CEP}}\right) \simeq(114,965)[\mathrm{MeV}]$ is represented as a star in Fig. 1. These results are essentially the same as the previously obtained results [23], and will be compared with the results in the following subsections.

\section{B. Imaginary number density in the PNJL model}

Now let us evaluate the imaginary number density $n_{q I}$ at the pure imaginary chemical potential from Eq. (14). The momentum integrations in Eqs. (16)-(18) are calculated with the Gaussian quadrature method. Figure 2 shows the 


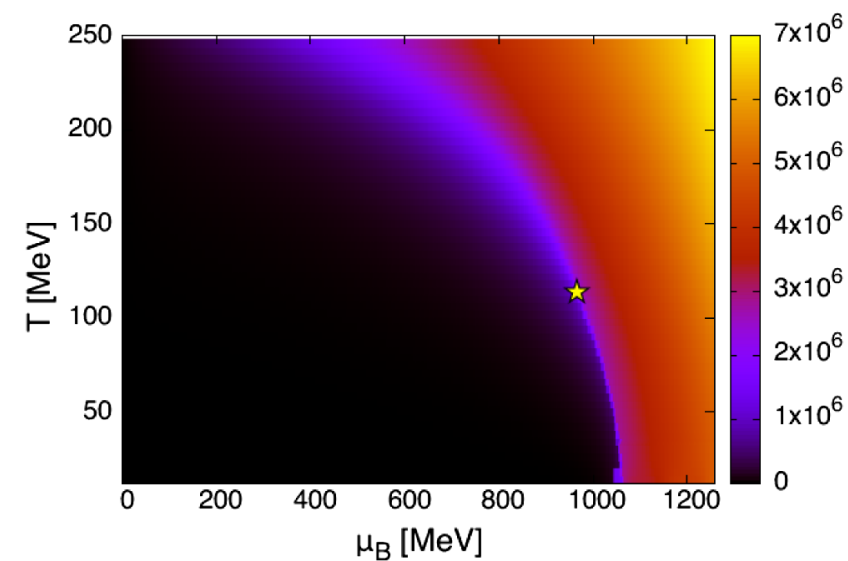

FIG. 1. The temperature and chemical potential dependences of the baryon number density $n_{B}\left[\mathrm{MeV}^{3}\right]$ in the PNJL model. The star is the critical endpoint $(\mathrm{CEP})\left(T^{\mathrm{CEP}}, \mu_{B}^{\mathrm{CEP}}\right) \simeq(114,965)[\mathrm{MeV}]$.

$\theta\left(=\mu_{I} / T\right)$ dependence of the imaginary number density. $n_{q I} / T^{3}$ are calculated at 161 values of $\mu_{I}$ for various temperatures. Because the PNJL model has the $Z_{3}$ symmetry and an anti-symmetry, $n_{q I}(\theta)=n_{q I}(\theta+2 \pi / 3)$ and $n_{q I}(\theta)=-n_{q I}(-\theta)$, we only show the region $0 \leq \theta \leq \pi / 3$ in Fig. 2. From Fig. 2, we find that $n_{q I}$ is well approximated by the Fourier series

$$
\frac{n_{q I}}{T^{3}}(\theta)=\sum_{k=1}^{N_{\text {sin }}} f_{3 k} \sin (3 k \theta),
$$

which is used instead of Eq. (4) since $f_{k}$ for $\bmod (k, 3) \neq 0$ are zero due to the $Z_{3}$ symmetry. As long as we are interested in the confinement phase of QCD here, the $Z_{3}$ symmetric feature in Eq. (19) remains intact. The obtained coefficients $f_{3 k}$ are listed in Table I.

\section{C. $N_{\max }$ dependence of the number density in the PNJL model}

Next, we calculate the grand canonical partition function at pure imaginary chemical potential with the integration

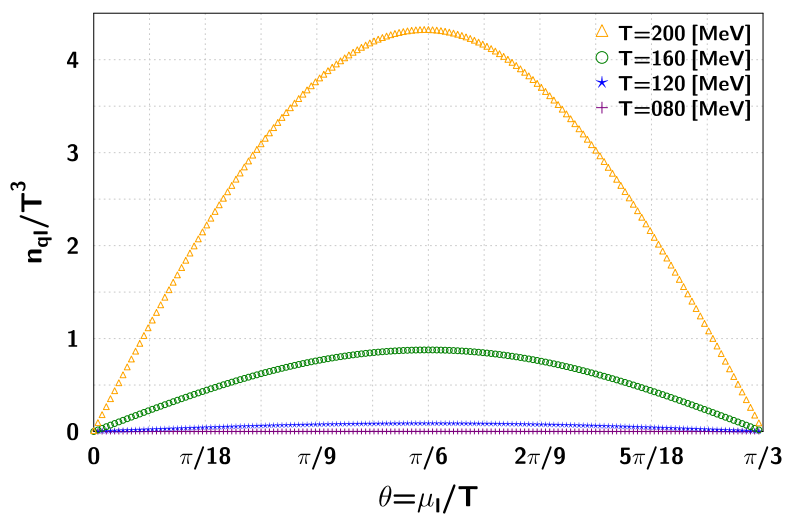

FIG. 2. The $\theta$ dependence of the imaginary number density in the PNJL model.
TABLE I. The coefficients $f_{3 k}$ from the data of $n_{q I} / T^{3}$ for each temperature.

\begin{tabular}{lcccc}
\hline \hline$T[\mathrm{MeV}]$ & $f_{3}$ & $f_{6}$ & $f_{9}$ & $f_{12}$ \\
\hline 200 & $2.2 \times 10^{-2}$ & $1.7 \times 10^{-4}$ & $1.9 \times 10^{-6}$ & $2.2 \times 10^{-8}$ \\
160 & $5.5 \times 10^{-3}$ & $9.0 \times 10^{-6}$ & $2.1 \times 10^{-8}$ & $5.8 \times 10^{-11}$ \\
120 & $7.2 \times 10^{-4}$ & $9.9 \times 10^{-8}$ & $2.0 \times 10^{-11}$ & $4.7 \times 10^{-15}$ \\
80 & $1.4 \times 10^{-5}$ & $1.7 \times 10^{-11}$ & $3.0 \times 10^{-17}$ & $\cdots$ \\
\hline \hline
\end{tabular}

method in Eq. (5). Here, the finite volume effect is included as the coefficient $V$ in Eq. (5), although the imaginary number densities and $f_{3 k}$ in Eq. (19) are computed by the formula for the infinite volume. In this paper, since we study the $N_{\max }$ and $N_{\text {sin }}$ dependences of the canonical approach, we use $V=(6[\mathrm{fm}])^{3}$ to minimize the finite $V$ effect, which is justified in comparison with the argument of Ref. [30], where $V \sim(5[\mathrm{fm}])^{3}$ is shown to be sufficiently large.

By performing Fourier transforms in Eq. (2) with 8,192 significant digits in decimal notation, we obtain the canonical partition functions. Finally, we can reconstruct the grand canonical partition function,

$$
Z_{\mathrm{GC}}(\mu, T, V)=\sum_{n=-N_{\max }}^{N_{\max }} Z_{C}(n, T, V) \xi^{n},
$$

where $N_{\max }$ is a maximum value of fluctuation of the net quark number in the system. Although theoretically, $N_{\max }$ should be taken infinity, practically in numerical calculations it is set at a finite value.

In Fig. 3, we present the $N_{\max }$ dependence of the baryon number density $n_{B}$ obtained from the canonical approach at $T=80[\mathrm{MeV}]$. The solid line is the exact number density calculated at the real chemical potential. The figure shows

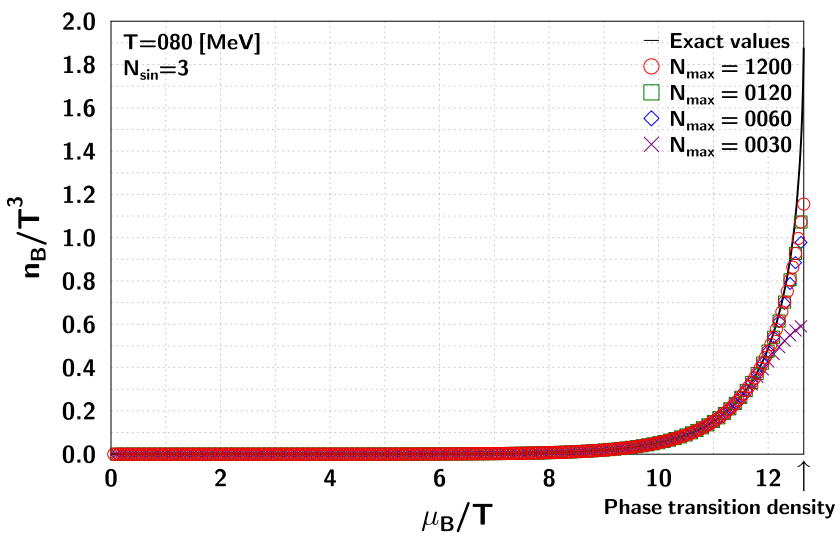

FIG. 3. The $N_{\max }$ dependence of the number density in the PNJL model. The solid line is the exact number density calculated at the real chemical potential. The other symbols are the number densities obtained from the canonical approach for several $N_{\max }$. 


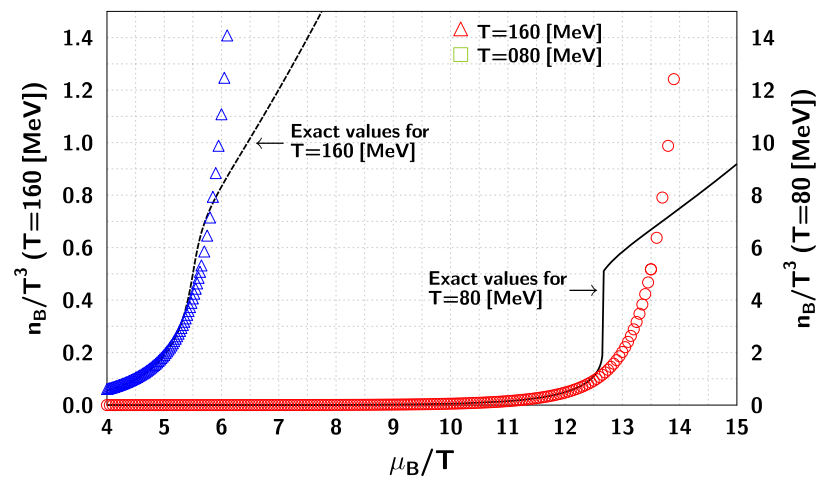

FIG. 4. The number densities around the phase transition or the crossover densities in the PNJL model. The solid and dashed lines are the exact number densities calculated at the real chemical potentials at $T=80$ and $160[\mathrm{MeV}]$, respectively. The open circles and open triangles are the number densities obtained from the canonical approach with $\left(N_{\max }, N_{\text {sin }}\right)=(1200,3)$ and $(1200,4)$ at $T=80$ and $160[\mathrm{MeV}]$, respectively.

numerical results only up to the point of phase transition density because the Fourier transforms in the canonical approach are no longer effective beyond the phase transition point. Actually, in Fig. 4, we can see that the canonical approach is ineffective beyond the phase transition point. From Fig. 3, we find that the behavior of the number density converges for $N_{\max }=120$ and larger. Note that the difference between $n_{B}$ calculated from the canonical approach and the exact values near the phase transition density comes from the finite $N_{\text {sin }}$ effect, which we discuss in the next subsection. Now we can understand the converging behavior of $n_{B}$ by comparing $N_{\max } /(3 V)=$ $120 /\left(3 \times 6^{3}\right) \sim 0.19\left[\mathrm{fm}^{-3}\right]$ with the normal nuclear matter density $0.17\left[\mathrm{fm}^{-3}\right]$. It is reasonable to expect that the fluctuations of the number density are in the same order of the nuclear matter density in the region of the chemical potential and temperature that we are looking at now.

\section{D. $N_{\sin }$ dependence of the number density in the PNJL model}

In this subsection, we discuss the $N_{\sin }$ dependence by using a sufficiently large number $N_{\max }=1200$ to suppress possible uncertainties due to finite $N_{\max }$. In Fig. 5, we show the $N_{\text {sin }}$ dependence of the baryon number density at $T=80,160$ and $200[\mathrm{MeV}]$. As in the case of Fig. 3, the figures show numerical results only up to the point of phase transition or crossover densities. The reason that the plot range is restricted to the lower density side up to a certain maximum point for $T>T^{\mathrm{CEP}}$ is that as shown in Fig. 4, the gradient of the number densities obtained from the canonical approach for $T=160[\mathrm{MeV}]$ monotonically increases and the canonical approach becomes ineffective beyond the crossover density. The solid lines are the exact number densities $n_{B}^{\text {exact }}$ calculated at the real chemical potential. The symbols represent the number densities obtained from the canonical approach, $n_{B}^{\text {canonical }}$. As $N_{\text {sin }}$ increases, the difference between $n_{B}^{\text {exact }}$ and $n_{B}^{\text {canonical }}$ becomes smaller.

In Fig. 6, we show the $N_{\text {sin }}$ dependence of the ratio of $n_{B}^{\text {canonical }}$ to $n_{B}^{\text {exact }}$ at $T=80,160$, and $200[\mathrm{MeV}]$. In this paper, to discuss the accuracy of the approximation, we set the effective region of the canonical approach as the region where the difference is less than $10 \%, 0.9<$ $n_{B}^{\text {canonical }} / n_{B}^{\text {exact }}<1.1$. For $N_{\text {sin }}=1$ at $T=80,160$, and $200[\mathrm{MeV}]$, the boundaries between the effective and ineffective regions of the canonical approach appear at the $89 \%, 74 \%$, and $65 \%$ of the phase transition or crossover densities, respectively. It turns out that as the temperature decreases, the Fourier series approximation with $N_{\sin }=1$ becomes better. These observations for $N_{\sin }=1$ are useful when actual lattice study is performed. For $N_{\sin }=3$ at $T=$ $80[\mathrm{MeV}]$ and $N_{\text {sin }}=4$ at $T=160[\mathrm{MeV}]$, we can reconstruct the exact baryon number density from the canonical approach up to the densities of (97-98)\% of the phase transition or crossover density within $10 \%$ accuracy. Moreover, for $N_{\text {sin }}=4$ at $T=200[\mathrm{MeV}], n_{B}^{\text {canonical }}$ can be computed with difference only less than $1.8 \%$ from the exact value until the crossover density.

In Fig. 7, we plot the boundaries between the effective and ineffective regions of the canonical approach for various $N_{\sin }$ and temperatures. Due to the definition of the (in)effective regions, in the left regions of the boundaries, $n_{B}$ can be computed in the accuracy $0.9<n_{B}^{\text {canonical }} / n_{B}^{\text {exact }}<1.1$. In general the boundary deviates
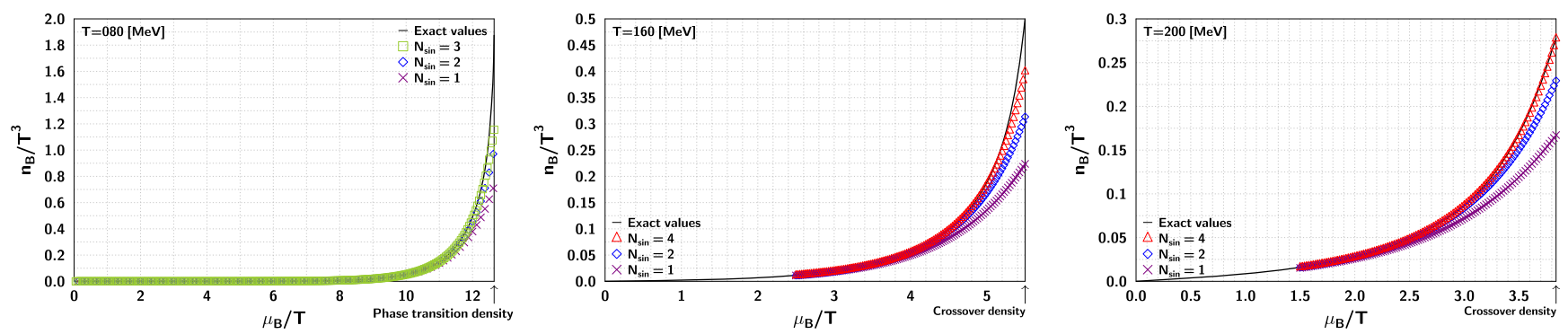

FIG. 5. The $N_{\text {sin }}$ dependence of $n_{B} / T^{3}$ in the PNJL model. The solid lines are the exact number densities calculated at the real chemical potential. 

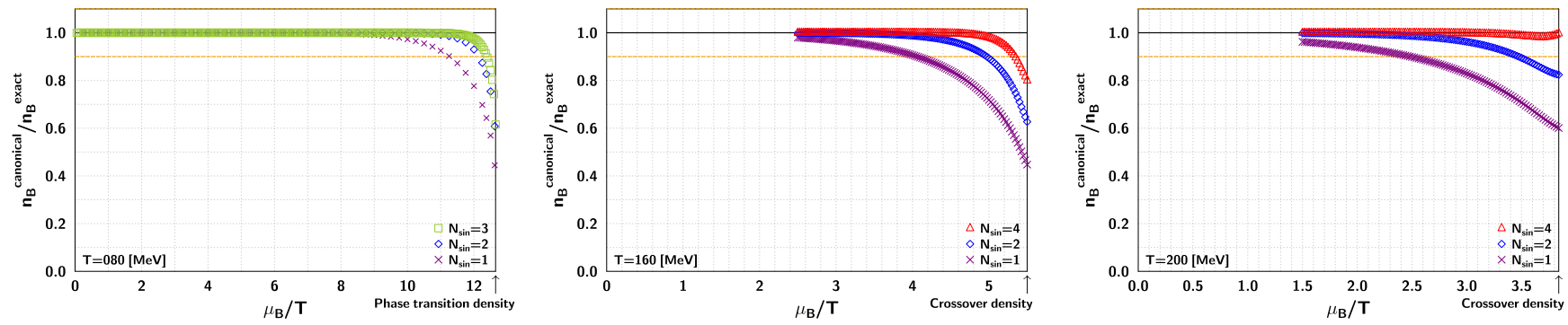

FIG. 6. The $N_{\text {sin }}$ dependence of $n_{B}^{\text {canonical }} / n_{B}^{\text {exact }}$ in the PNJL model. $n_{B}^{\text {canonical }}$ is the number density obtained from the canonical approach and $n_{B}^{\text {exact }}$ is the exact number density calculated at the real chemical potential. The solid and dashed lines represent the exact value $\left(n_{B}^{\text {canonical }} / n_{B}^{\text {exact }}=1.0\right)$ and the $10 \%$ difference values $\left(n_{B}^{\text {canonical }} / n_{B}^{\text {exact }}=0.9\right.$ and 1.1$)$.

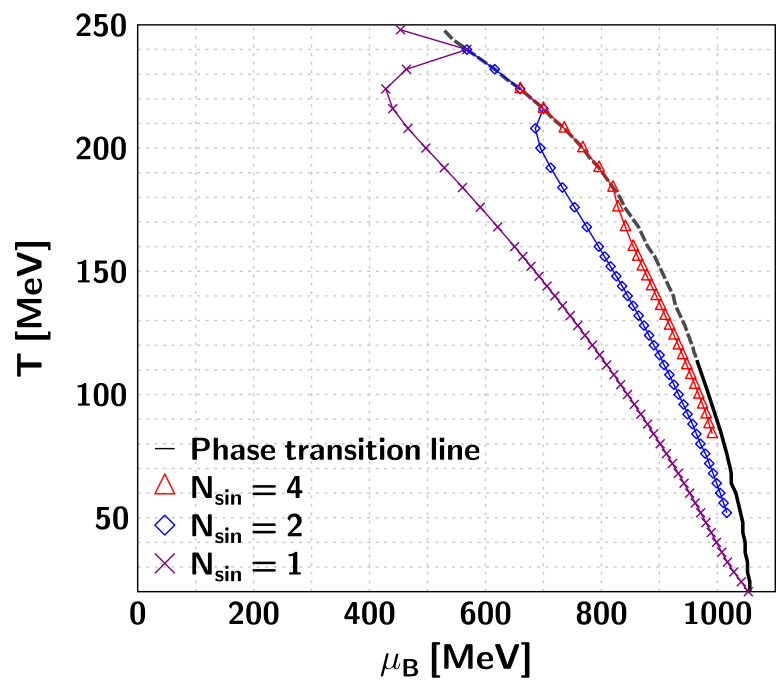

FIG. 7. The boundaries between the effective and ineffective regions of the canonical approach for $N_{\text {sin }}$ in the PNJL model. The black solid and dashed lines represent the first-order phase transition and crossover lines, respectively. The points of making a $10 \%$ difference between the exact $n_{B}$ and the results from the canonical approach are plotted. We plot the symbols on the crossover line when the difference is less than $10 \%$ in the confinement phase.

from the phase transition or crossover line. For $N_{\text {sin }}=2$ and 4, the boundaries reach the crossover line that is plotted. For instance, for $N_{\text {sin }}=4$, this occurs at $T=(184-224)[\mathrm{MeV}]$. The reason is that there is no crossover or phase transition structure in the Fourier series approximation with finite $N_{\text {sin }}$ since the function is analytic. From Fig. 7, we find that most of the confinement phase can be reliably studied by the canonical approach with $N_{\text {sin }}=4$. Furthermore, for $T<T^{\mathrm{CEP}}$ and $\mu_{B}<900[\mathrm{MeV}], N_{\text {sin }}=1$ or 2 is enough to reconstruct the exact number density from the canonical approach. The results suggest that the application of the canonical approach to the lattice QCD is useful, especially in the confinement phase.

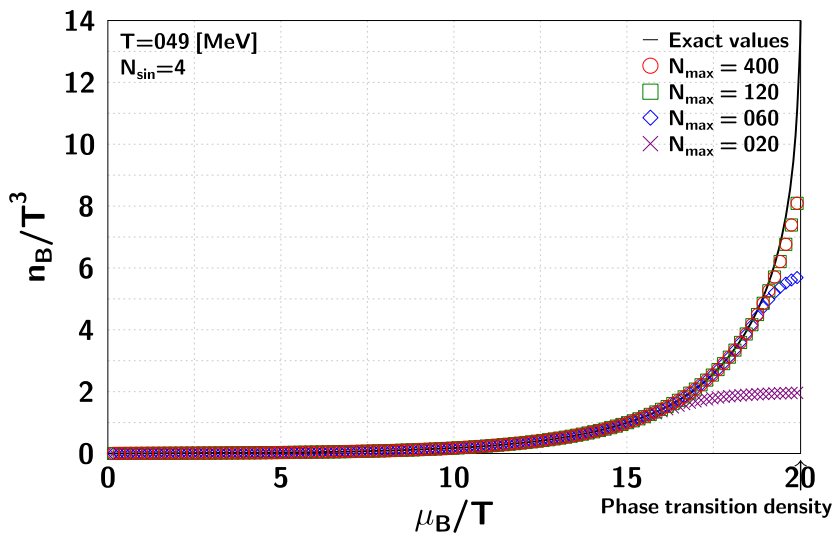

FIG. 8. The $N_{\max }$ dependence of the number density in the NJL model. The solid line is the exact number density calculated at the real chemical potential. The other symbols are the number densities obtained from the canonical approach for several $N_{\max }$.

\section{E. Comparison with the NJL and PNJL models}

At the end of this section, we consider the model dependence by comparing the results of the PNJL model with those of the NJL one. In the NJL model, we obtain the coefficients $f_{k}$ from 161 values of data of $n_{q I} / T^{3}$ such as Table II. Here, we use not Eq. (19) but Eq. (4) since the NJL model does not have the $Z_{3}$ symmetry. As it was done in the PNJL model, we set $V$ in Eq. (3) to $(6[\mathrm{fm}])^{3}$ and reconstruct the grand canonical partition function by performing the Fourier transforms with 8,192 significant digits in decimal notation.

TABLE II. The coefficients $f_{k}$ from the data of $n_{q I} / T^{3}$ for each temperature in the NJL model.

\begin{tabular}{lcccc}
\hline \hline$T[\mathrm{MeV}]$ & $f_{1}$ & $f_{2}$ & $f_{3}$ & $f_{4}$ \\
\hline 79 & $2.7 \times 10^{-1}$ & $2.3 \times 10^{-3}$ & $2.9 \times 10^{-5}$ & $4.2 \times 10^{-7}$ \\
49 & $3.7 \times 10^{-2}$ & $1.8 \times 10^{-5}$ & $1.3 \times 10^{-8}$ & $1.1 \times 10^{-11}$ \\
29 & $6.5 \times 10^{-4}$ & $1.9 \times 10^{-9}$ & $7.9 \times 10^{-15}$ & $3.9 \times 10^{-20}$ \\
\hline \hline
\end{tabular}



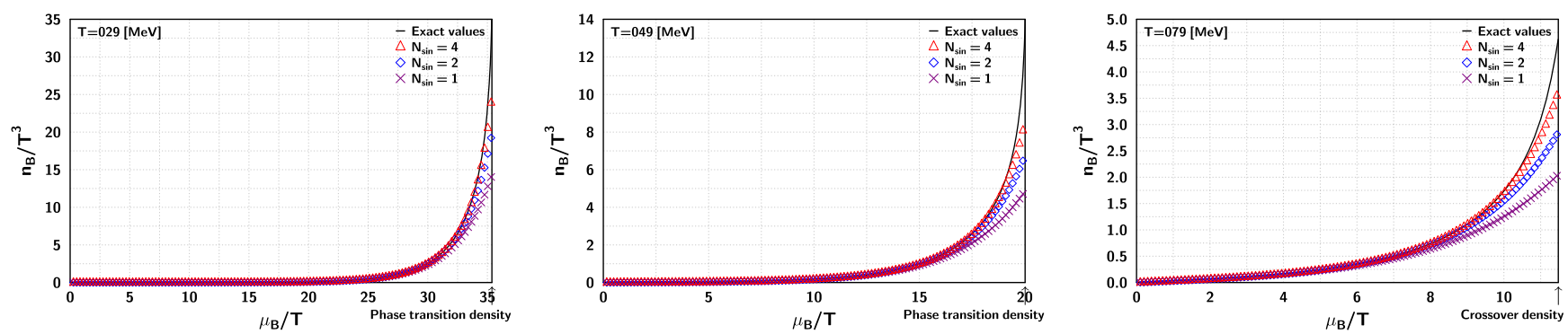

FIG. 9. The $N_{\sin }$ dependence of $n_{B} / T^{3}$ in the NJL model. The solid lines are the exact number densities calculated at the real chemical potential.
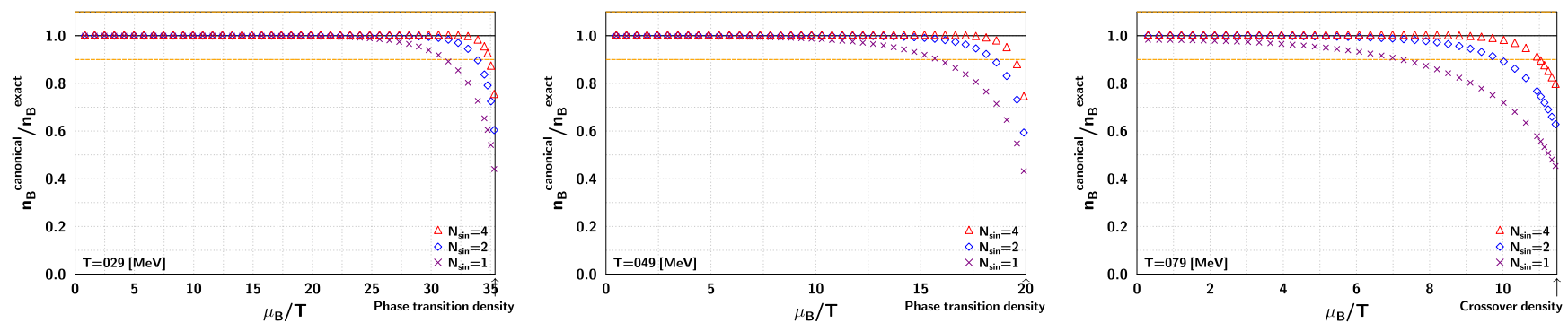

FIG. 10. The $N_{\text {sin }}$ dependence of $n_{B}^{\text {canonical }} / n_{B}^{\text {exact }}$ in the NJL model. $n_{B}^{\text {canonical }}$ is the number density obtained from the canonical approach and $n_{B}^{\text {exact }}$ is the exact number density calculated at the real chemical potential.

Figure 8 shows the $N_{\max }$ dependence of the baryon number density at $T=49[\mathrm{MeV}]$ in the NJL model. The solid line is the exact number density calculated at the real chemical potential. We find that the behavior of the number density converges for $N_{\max }=120$ and larger, which is the

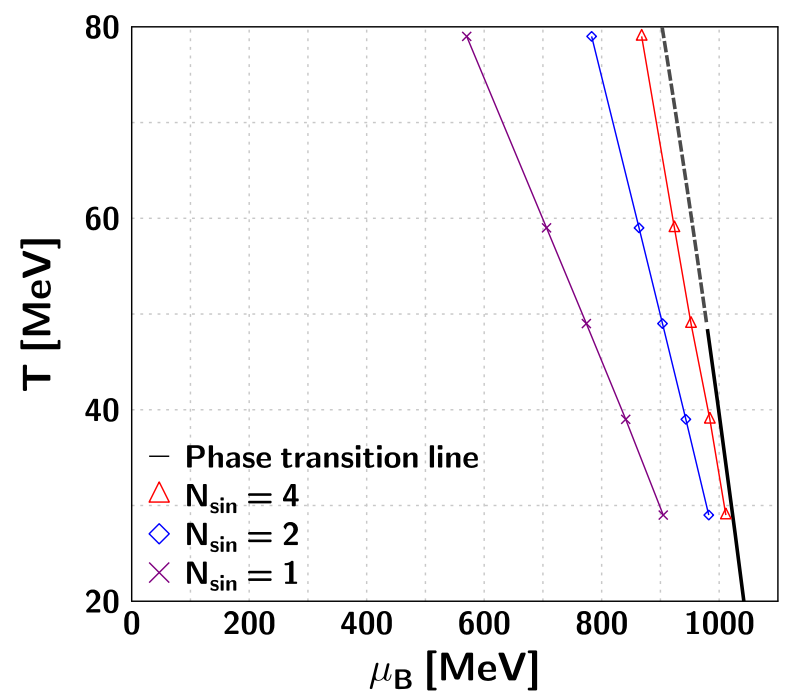

FIG. 11. The boundaries between the effective and ineffective regions of the canonical approach for $N_{\text {sin }}$ in the NJL model. The black solid and dashed lines represent the first-order phase transition and crossover lines, respectively. The points of making a $10 \%$ difference between the exact $n_{B}$ and the results from the canonical approach are plotted. same as the result of the PNJL model. In the following discussion for the NJL model, we use $N_{\max }=400$.

In Fig. 9, we show the $N_{\text {sin }}$ adependence of the number density at $T=29,49$, and $79[\mathrm{MeV}]$ in the NJL model. The solid lines are the exact number densities $n_{B}^{\text {exact }}$ calculated at the real chemical potential. The symbols represent the number densities obtained from the canonical approach, $n_{B}^{\text {canonical }}$. As $N_{\text {sin }}$ increases, the difference between $n_{B}^{\text {exact }}$ and $n_{B}^{\text {canonical }}$ becomes small.

In Fig. 10, we show the $N_{\text {sin }}$ dependence of the ratio of $n_{B}^{\text {canonical }}$ to $n_{B}^{\text {exact }}$ in the NJL model. For $N_{\text {sin }}=4$ at $T=29$, 49 , and $79[\mathrm{MeV}]$, we can reconstruct the exact baryon number density from the canonical approach up to the densities of $99 \%, 97 \%$, and $96 \%$ of the phase transition or crossover density within $10 \%$ accuracy, respectively.

In Fig. 11, we plot the symbols on the high-density limits of the effective region of the canonical approach for each $N_{\text {sin }}$ and temperature in the NJL model. We find that the effective region of the canonical approach for $N_{\sin }=4$ can cover in most of the confinement phase. For $T \lesssim 49 \sim$ $T^{\mathrm{CEP}}[\mathrm{MeV}]$ and $\mu_{B} \lesssim 900[\mathrm{MeV}], N_{\text {sin }}=1$ or 2 is enough to reconstruct the exact number density from the canonical approach. The results have universality for at least the NJL and PNJL models.

\section{SUMMARY}

We have investigated the effective region in the phase diagram where the canonical approach in the NJL and 
PNJL models works. We have calculated the 161 data of the imaginary number densities as functions of the pure imaginary chemical potential. By using the integration method of a Fourier series with finite $N_{\text {sin }}$ for the imaginary number densities and performing Fourier transforms with the multiple-precision arithmetic, we have reconstructed the grand canonical partition function, which is written as a fugacity expansion with finite $N_{\max }$. After that, we have calculated the number densities at the real chemical potential from the grand canonical partition function. Because the number densities are already known in the NJL and PNJL models, we can clarify the region where the canonical approach works well by comparing the number densities obtained from the canonical approach with the exact ones.

We have shown the $N_{\max }$ and $N_{\text {sin }}$ dependences of the number densities obtained from the canonical approach in each model. In the investigation of the $N_{\max }$ dependence, we have found that the finite $N_{\max }$ effect for the number density is suppressed for the maximum value of the fluctuation of the net quark number density in the system, $N_{\max } / V$, larger than $0.56\left[\mathrm{fm}^{-3}\right]$.

For the $N_{\text {sin }}$ dependence, we have found that the results for $N_{\sin }$ up to 4 can reconstruct the number density from the canonical approach up to the densities of $96 \%$ of the phase transition or crossover density within $10 \%$ accuracy. Moreover, $N_{\text {sin }}=1$ or 2 is enough to reconstruct the exact number density within $10 \%$ accuracy for $T<T^{\mathrm{CEP}}$ and
$\mu_{B}<900[\mathrm{MeV}]$. The results have universality for at least the NJL and PNJL models. They suggest that the application of the canonical approach to the lattice QCD is useful, especially in the confinement phase.

In this paper, we have discussed the effective region of the canonical approach for the number density in the NJL and PNJL models. It remains to be investigated for other physical quantities and other models.

\section{ACKNOWLEDGMENTS}

This work was supported by the National Research Foundation of Korea (NRF) grant funded by the Korean government (MSIT) (No. 2018R1A5A1025563). The work of S.-i. N. is also supported in part by the NRF fund (No. 2019R1A2C1005697). A. H. is supported in part by Japan Society for the Promotion of Science for Grants-inAid for Scientific Research (No. JP17K05441 (C)) and for Scientific Research on Innovative Areas (No. 18H05407). This work was supported by "Joint Usage/Research Center for Interdisciplinary Large-scale Information Infrastructures" (Project ID: jh190051-NAH and jh200013-NAH) and "High Performance Computing Infrastructure" in Japan. The calculations were carried out on SX-ACE and OCTOPUS at RCNP/CMC of Osaka University.
[1] A. Hasenfratz and D. Toussaint, Canonical ensembles and nonzero density quantum chromodynamics, Nucl. Phys. B371, 539 (1992).

[2] A. Alexandru, M. Faber, I. Horvath, and K. F. Liu, Lattice QCD at finite density via a new canonical approach, Phys. Rev. D 72, 114513 (2005).

[3] S. Kratochvila and P. de Forcrand, The Canonical approach to finite density QCD, Proc. Sci., LAT2005 (2006) 167 [arXiv:hep-lat/0509143].

[4] P. de Forcrand and S. Kratochvila, Finite density QCD with a canonical approach, Nucl. Phys. B, Proc. Suppl. 153, 62 (2006).

[5] S. Ejiri, Canonical partition function and finite density phase transition in lattice QCD, Phys. Rev. D 78, 074507 (2008).

[6] A. Li, A. Alexandru, K. F. Liu, and X. Meng, Finite density phase transition of QCD with $N_{f}=4$ and $N_{f}=2$ using canonical ensemble method, Phys. Rev. D 82, 054502 (2010).

[7] A. Li, A. Alexandru, and K. Liu, Critical point of $N_{f}=3$ QCD from lattice simulations in the canonical ensemble, Phys. Rev. D 84, 071503 (2011).

[8] J. Danzer and C. Gattringer, Properties of canonical determinants and a test of fugacity expansion for finite density lattice QCD with Wilson fermions, Phys. Rev. D 86, 014502 (2012).

[9] K. Morita, V. Skokov, B. Friman, and K. Redlich, Net baryon number probability distribution near the chiral phase transition, Eur. Phys. J. C 74, 2706 (2014).

[10] C. Gattringer and H. P. Schadler, Generalized quark number susceptibilities from fugacity expansion at finite chemical potential for $N_{f}=2$ Wilson fermions, Phys. Rev. D 91, 074511 (2015).

[11] R. Fukuda, A. Nakamura, and S. Oka, Canonical approach to finite density QCD with multiple precision computation, Phys. Rev. D 93, 094508 (2016).

[12] A. Nakamura, S. Oka, and Y. Taniguchi, QCD phase transition at real chemical potential with canonical approach, J. High Energy Phys. 02 (2016) 054.

[13] D. L. Boyda, V. G. Bornyakov, V. A. Goy, V. I. Zakharov, A. V. Molochkov, A. Nakamura, and A. A. Nikolaev, Novel approach to deriving the canonical generating functional in lattice QCD at a finite chemical potential, Pis'ma Zh. Eksp. Teor. Fiz. 104, 673 (2016) [JETP Lett. 104, 657 (2016)].

[14] V. A. Goy, V. Bornyakov, D. Boyda, A. Molochkov, A. Nakamura, A. Nikolaev, and V. Zakharov, Sign problem in finite density lattice QCD, Prog. Theor. Exp. Phys. 2017, 031D01 (2017). 
[15] V. G. Bornyakov, D. L. Boyda, V. A. Goy, A. V. Molochkov, A. Nakamura, A. A. Nikolaev, and V. I. Zakharov, New approach to canonical partition functions computation in $N_{f}=2$ lattice QCD at finite baryon density, Phys. Rev. D 95, 094506 (2017).

[16] D. Boyda, V. G. Bornyakov, V. Goy, A. Molochkov, A. Nakamura, A. Nikolaev, and V. I. Zakharov, Lattice QCD thermodynamics at finite chemical potential and its comparison with experiments, arXiv:1704.03980.

[17] M. Wakayama, V. G. Borynakov, D. L. Boyda, V. A. Goy, H. Iida, A. V. Molochkov, A. Nakamura, and V. I. Zakharov, Lee-Yang zeros in lattice QCD for searching phase transition points, Phys. Lett. B 793, 227 (2019).

[18] M. Wakayama and A. Hosaka, Search of QCD phase transition points in the canonical approach of the NJL model, Phys. Lett. B 795, 548 (2019).

[19] Y. Nambu and G. Jona-Lasinio, Dynamical model of elementary particles based on an analogy with superconductivity. 1., Phys. Rev. 122, 345 (1961).

[20] Y. Nambu and G. Jona-Lasinio, Dynamical model of elementary particles based on an analogy with superconductivity. II, Phys. Rev. 124, 246 (1961).

[21] T. Kunihiro, Quark number susceptibility and fluctuations in the vector channel at high temperatures, Phys. Lett. B 271, 395 (1991).
[22] T. Hatsuda and T. Kunihiro, QCD phenomenology based on a chiral effective Lagrangian, Phys. Rep. 247, 221 (1994).

[23] K. Fukushima, Chiral effective model with the Polyakov loop, Phys. Lett. B 591, 277 (2004).

[24] S. Roessner, T. Hell, C. Ratti, and W. Weise, The chiral and deconfinement crossover transitions: PNJL model beyond mean field, Nucl. Phys. A814, 118 (2008).

[25] X. F. Meng, A. Li, A. Alexandru, and K. F. Liu, Winding number expansion for the canonical approach to finite density simulations, Proc. Sci., LATTICE2008 (2008) 032 [arXiv:0811.2112].

[26] M. D'Elia and F. Sanfilippo, Thermodynamics of two flavor QCD from imaginary chemical potentials, Phys. Rev. D 80, 014502 (2009).

[27] T. Takaishi, P. de Forcrand, and A. Nakamura, Equation of state at finite density from imaginary chemical potential, Proc. Sci., LAT2009 (2009) 198 [arXiv:1002.0890].

[28] V. Skokov, B. Friman, and K. Redlich, Quark number fluctuations in the Polyakov loop-extended quark-meson model at finite baryon density, Phys. Rev. C 83, 054904 (2011).

[29] D. M. Smith, Multiple precision computation, FMLIB1.3 (2015), http://myweb.lmu.edu/dmsmith/FMLIB.html.

[30] K. Xu and M. Huang, Zero-mode contribution and quantized first order phase transition in a droplet quark matter, Phys. Rev. D 101, 074001 (2020). 